Max-Planck-Institut für demografische Forschung

Max Planck Institute for Demographic Research

Doberaner Strasse 114 - D-18057 Rostock - GERMANY

Tel +49 (0) 3812081 - 0; Fax +49 (0) 3812081 - 202;

http://www.demogr.mpg.de

MPIDR WORKING PAPER WP 2002-041

SEPTEMBER 2002

\title{
Daily activities and survival at older ages
}

Petra L. Klumb (petra.klumb@tu-berlin.de)

Heiner Maier (maier@ demogr.mpg.de)

This working paper has been approved for release by: James W. Vaupel (jwv@ demogr.mpg.de)

Head of the Laboratory of Survival and Longevity.

(C) Copyright is held by the authors.

Working papers of the Max Planck Institute for Demographic Research receive only limited review.

Views or opinions expressed in working papers are attributable to the authors and do not necessarily reflect those of the Institute. 
DAILY ACTIVITIES AND SURVIVAL AT OLDER AGES*

PETRA L. KLUMB

Technische Universität Berlin, Germany

HEINER MAIER

Max Planck Institute for Demographic Research, Rostock, Germany

Running Head: ACTIVITIES AND SURVIVAL

Key Words: Daily activities, mortality, old age, production, Berlin Aging Study 6028 words, 3 tables, 2 figures 


\section{DAILY ACTIVITIES AND SURVIVAL AT OLDER AGES}

\section{ABSTRACT}

This study tested the hypothesis that time spent on regenerative (e.g., resting), productive (e.g., housework), and consumptive activities (e.g., meeting friends) is associated with survival in persons aged 70 and older. An observational study with semi-annual mortality follow-ups was carried out in the former West Berlin, Germany. The sample was stratified by age and sex and consisted of 473 persons aged 70 to 103 years. Study participants lived in the community as well as in institutions. Activity measures were assessed in 1990-1993 by structured interviews in the participants' homes. Cox regression was used to model survival from time of interview. The main outcome measure was survival on 3 February 2000. Consumptive activities were related to survival (relative risk $=0.76,95 \%$ confidence interval 0.58 to 1.00 ) after several confounding factors were controlled for. There were indications that the greatest survival benefit is achieved with a medium amount of time devoted to consumptive activities. Our results support the idea that daily activities are linked to survival via a psychosocial pathway, which might involve perceived quality of life. Consumptive activities (e.g., meeting friends, reading a novel) may contribute considerably to maintaining health and achieving longevity, because they are performed on a daily basis and their effects may accumulate over the life course. 


\section{DAILY ACTIVITIES AND SURVIVAL AT OLDER AGES}

Understanding the pattern in determinants of late-life survival becomes increasingly important as the population ages (Christensen and Vaupel, 1996). It is well known that healthrelated behaviors such as smoking, drinking and physical activity are related to mortality and survival. There are also reports suggesting that other daily activities such as meeting friends, shopping, and doing volunteer work are associated with a longer life (Glass et al. 1999; Lennartsson and Silverstein 2001; Musick et al. 1999). The etiologic mechanisms relating these daily activities to length of life are not well understood. Both physical (Riddoch 2000) and psychosocial pathways (Rowe and Kahn 1998; Thoits 1983) have been proposed. For example, the physical component of activities such as hanging up laundry or house cleaning exerts a direct influence on bodily processes. It improves musculo-skeletal strength as well as cardiopulmonary performance, and it may thereby reduce the risk of cardiovascular disease (Helmert, Herman, \& Shea, 1994). Physical activity also influences lipid and glucose metabolism as well as lipoprotein levels beneficially (Goldberg \& Elliot, 1985).

In addition to physical influences, psychosocial effects also are thought to be in operation. Engagement in challenging activities such as solving puzzles or reading a novel affords learning and enjoyment (Baltes 1996). Moreover, social activities such as chatting or playing board games may create a sense of belonging and emotional closeness. Involvement in this kind of activity has two consequences. There is a direct effect on well-being (Clark and Watson 1988) and a stress-buffering effect (House et al. 1988).

Previous research on activities and survival has been hampered by the lack of a general theory which provides a well-defined conceptualization of activity types and their antecedents and consequences. Basing our study on Baltes’ two-component model (Baltes et al. 1999), we distinguish two broad categories of daily activities (see Figure 1). On the one hand, there are regenerative activities that have to be carried out by physiological necessity (e.g., bathing, 
eating, and resting). On the other hand, there are activities that can be selected more or less deliberately on the basis of individual abilities and preferences. The third-party criterion (Reid 1934) serves to further sub-divide this optional category into productive and consumptive activities. If an activity is performed predominantly due to its outcomes and can, therefore, be delegated to a third party without losing its beneficial effect (e.g., doing laundry, house cleaning, running errands), then it is productive. In industrialized societies individuals of all ages delegate at least some productive activities to third parties, for example, when they purchase market products and services instead of producing them at home. In contrast, if an activity is performed primarily for its own sake and cannot, therefore, be delegated to a third party without losing benefit (e.g., meeting friends, reading a novel, watching TV), then it is consumptive.

Two different types of benefits are connected with productive and consumptive activities. Benefits obtained from productive activities are mainly mediated through their outcomes. Psychosocial and health benefits accruing from productive activities can thus be maintained to a large extent by delegating them. Benefits accruing from consumptive activities, in contrast, are more direct and result from the process of performing the activityin the microeconomic literature they are called process benefits (Juster and Stafford 1991). Process benefits cannot be maintained by delegation of the activity to a third party. Consequently, we hypothesized that not engaging in consumptive activities has more damaging consequences for well-being, health, and longevity than not engaging in productive activities.

Figure 1 about here 
Some previous studies on activities and survival adopted the assumption that health benefits increase monotonically with increasing participation in a particular activity. Alternatively, the microeconomic concept of "decreasing returns on investment" has been used to describe the relationship between daily activities and health outcomes (Bird and Fremont 1991). According to this concept, the relationship may level off beyond a certain point because additional time units allocated to an activity may not make any difference and may even be associated with decreasing benefits due to resource depletion. For instance, once a person has reached a state of relaxation, more time allocated to reading, playing games, or watching TV may not improve this state any further. Thus, we hypothesized that a medium amount of time devoted to consumptive activities conveys the greatest survival benefit.

In sum, we examined two hypotheses. First, we expected that consumptive, but not regenerative or productive activities, are associated with survival. Second, we expected that benefits arising from consumptive activities are greatest for a medium duration of the activity, while a very long or a very short duration is associated with smaller benefits.

\section{METHOD}

\section{Participants and Sampling}

We used data from the first measurement occasion of the Berlin Aging Study (Baltes and Mayer 1999), which took place in the time period 1990-1993. The study was designed to be representative of the West-Berlin population aged 70+, while oversampling men and the very old. Samples originated from a random draw of addresses from the general registry (Landeseinwohneramt) of West Berlin. To obtain the final sample of 516 individuals stratified by age and sex, a much larger number of addresses had to be drawn. The study design consists of a hierarchical sequence of four levels of participation, with increasing numbers of variables but decreasing numbers of participants at each consecutive level: (a) the verified parent 
sample $(\underline{\mathrm{N}}=1908)$; (b) the short-contact sample $(\underline{\mathrm{N}}=1264)$; (c) the Intake-Assessment sample ( $\underline{N}=928)$; and (d) the Intensive-Protocol sample $(\underline{N}=516)$ used in the present study with its 14 sessions of multidisciplinary assessment. Extensive selectivity analyses (Lindenberger et al. 1999) showed the Intensive-Protocol sample to be a somewhat positive selection of the parent sample. The magnitude of the selectivity effects was largest for general intelligence, but it did not exceed half a standard deviation for any of the analyzed domains of functioning. With one exception (dementia prevalence), selectivity effects did not interact with age or gender. Furthermore, comparisons of the sample with the Berlin Census Data showed no significant differences in indices such as marital status, proportion of institutionalized persons, and educational and income levels.

The Intensive-Protocol sample includes individuals ranging in age from 70 to 103 . The sample was stratified for age and sex, resulting in 43 women and 43 men in each of six age/cohort groups: 70-74 years (born 1915-1922), 75-79 years (born 1910-1917), 80-84 years (born 1905-1913), 85-89 years (born 1900-1908), 90-94 years (born 1896-1902), and 95-103 years (born 1883-1897). Based on judgements made by one of us (P.K.), we excluded 31 participants from our analyses due to implausible activity data. Most of these persons were diagnosed as suffering from dementia.

Mortality status information and the date of death for the deceased participants were obtained from the State Registry Office. Mortality information on twelve individuals could not be obtained because they had moved out of the Berlin area. These individuals were not considered in our analyses. This means that we utilized a total sample of 473 persons (230 women and 243 men) in our study.

\section{Measures}

Two types of measures were relevant: activity measures and a set of covariates. As covariates we rigorously chose common determinants of activity involvement and mortality 
(Rowe and Kahn 1998). The set of covariates included age, sex, years of education, measures of health and cognitive status, an indicator of whether or not participants lived with a spouse, and an indicator of whether or not they lived in an institution.

Activity measures. The "Yesterday Interview" (YI, Moss and Lawton 1982) was used to reconstruct the participants' day preceding the interview from waking up to falling asleep. In a separate study, we compared self-reports assessed with the YI to in-situ self-reports and found acceptable agreement (Klumb and Baltes 1999b). The YIs took place in the participants' homes and lasted an average of about 50 minutes. Interview data were first categorized into 44 activity codes. We quantified the levels of intercoder agreement with the kappa statistic (Cohen 1960). Kappas for all of the 44 activity codes were above 0.8 , suggesting high levels of intercoder agreement. The 44 activity codes were then condensed into 13 activity domains. Table 1 displays the average amount of time allocated to each of the 13 domains. Activity domains were then assigned to three broad activity categories: regenerative, productive, and consumptive activities (Klumb and Baltes 1999a).

Table 1 about here

Regenerative activities serve to maintain one's physical existence. This activity category comprised the activity domains "resting" and "self maintenance". For the distinction between productive and consumptive activities, we adopted the third-party criterion (Reid 1934), which allows for a clear-cut categorization of activity domains. The category "productive activities" resulted from collapsing the following five activity domains: "gardening", "helping others" (including volunteer work and provision of care for relatives), 
"housework" (including maintenance of home and possessions), "paid work", and "running errands". The six remaining domains were aggregated into the category "consumptive activities". Specifically, this category comprised the activity domains "active leisure" (such as attending adult education courses or performing sports), "health-related activities" (including visits to doctors), "locomotion" (such as walking, driving with own vehicle or riding as a passenger), "reading", "social interaction", and "watching TV/listening to radio/records/tapes". Locomotion and health-related activities belong to this category because it is impossible to delegate them to a third person. Locomotion in the context of shopping and errands, however, was coded as productive activity.

For some of the analyses, time spent on an activity (measured in minutes) was coded as either high or low, based on a median split (Table 2). The hypothesis of decreasing returns was addressed with the help of four strata $\left(<25^{\text {th }}\right.$ percentile; $\geq 25^{\text {th }}$ and $<50^{\text {th }}$ percentile; $\geq$ $50^{\text {th }}$ and $<75^{\text {th }}$ percentile $\geq 75^{\text {th }}$ percentile) reflecting differences in the amount of time spent on an activity (Table 3).

Education. We used the number of years spent in formal educational settings as an indicator of socio-economic status. In addition to the number of years spent in elementary school and the different types of high school in Germany (graduation after 10 to 13 years of schooling), this variable also includes formal professional (e.g., apprenticeships) and academic (e.g., university) training. On average, participants in this sample had 10.8 years of education $(\underline{\mathrm{SD}}=2.3)$.

Number of diagnoses. We selected the number of diagnosed moderate or severe illnesses as an externally assessed indicator of participants' general health status. Diagnoses were determined in the course of consensus conferences of the research physician and psychiatrist based on a standardized summary of clinical findings from all diagnostic procedures. Diagnosed moderate and severe illnesses were summed up to form the variable 
"number of diagnoses". On average, participants in this sample had 8 diagnoses of moderate or severe illnesses $(\underline{\mathrm{SD}}=4)$.

Balance / Gait. Functional health was represented by a unit-weighted composite of clinical assessments of balance and gait, the Romberg Stance, and the Turn 360 tasks (Tinetti 1986). In the Romberg Stance task, participants stood upright for about one minute, with legs as close together as possible, arms extended in front of the body, palms turned up, and eyes closed. Performance was scored by a physician on a six-point scale ranging from "no swaying" to "not able to stand upright at all." In the Turn 360 task, subjects were asked to perform a full turn around their body axis as fast as they could without risking a fall. The score corresponded to the number of steps needed to finish the circle. For the purpose of the present analyses the Balance/Gait composite was represented as a $\mathrm{z}$-score $(\underline{\mathrm{M}}=0, \underline{\mathrm{SD}}=1)$, with higher scores indicating higher levels of functional health.

Digit Letter test. We employed the Digit-Letter test, a measure of perceptual speed, as an indicator of cognitive functioning. On a large table visible throughout the whole procedure, each of nine different letters was assigned to a digit. Participants were presented with tables containing six digits and their task was to name the corresponding letters. The score consisted of the number of correct answers given within three minutes. Stimulus presentation and data collection were supported by a Macintosh SE30 personal computer equipped with a Micro Touch Systems touch-sensitive screen. For the purpose of the present analyses the Digit Letter test was represented as a $\mathrm{z}$-score $(\underline{\mathrm{M}}=0, \underline{\mathrm{SD}}=1)$, with higher scores reflecting higher levels of cognitive functioning.

Living with spouse. We included a dichotomous variable indicating whether or not a participant was living in the same household with his or her spouse or partner. This information was based on self-reporting by the participants and was verified by interviewers' 
observation. $\mathrm{N}=147$ persons (31 percent) lived with their spouse or partner in the same household, $\mathrm{N}=326$ (69 percent) did not.

Living in institution. We included an indicator reflecting whether a participant was living in the community or in an institution. This information was based on self-reporting by the participants and it was verified by interviewers' observation. $\mathrm{N}=409$ persons (86 percent) lived in the community, $\mathrm{n}=64$ (14 percent) lived in institutions.

Vital status. The vital status of participants in the Berlin Aging Study is monitored at regular intervals. By February 2000 (representing a 7 to 10 year period after baseline assessment), 318 individuals, or $67.2 \%$ of this sample, were registered in the state records as deceased, and 155 persons, or $32.8 \%$, were registered as living. As is to be expected for a sample of this advanced age, a larger proportion of the oldest old had died (older than 85 years: $\mathrm{n}=215$ decedents vs. $\mathrm{n}=15$ survivors) than in the younger age group $(70-84$ years: $\mathrm{n}=$ 103 decedents vs. $\mathrm{n}=140$ survivors). As is also to be expected, a larger proportion of men had died ( $\mathrm{n}=179$ decedents vs. $\mathrm{n}=64$ survivors $)$ than women $(\mathrm{n}=139$ decedents vs. $\mathrm{n}=91$ survivors).

Statistical Analyses

Cox proportional hazards regression models (Cox 1972) were evaluated for the effects of risk factors. We used the PHREG procedure (Allison 1995) from the SAS software package to estimate Cox regression models. We report relative risks and their $95 \%$ confidence intervals.

\section{RESULTS}

Figure 2 displays the average levels of regenerative, productive, as well as consumptive activities by sex and age group (70-84 vs. $85+$ ). Average levels of regenerative activities for men and women aged 70-84 were both about 4 hours and 20 minutes (less than a 
third of the waking day). For women and men aged 85+ they were about 6 hours and 40 minutes (45\% of the waking day). Women aged $70-84$ spent 3 hours and 40 minutes, on average, with productive activities (one fourth of their waking day), while the other groups allocated only 2 hours to this kind of activity. The average time dedicated to consumptive activities varied between 8 hours and 40 minutes (men aged 70-84) and 6 hours (women aged $85+$ ), with women aged 70-84 (7 hours and 30 minutes) and men aged 85+ (6 hours and 30 minutes) lying between these two extremes.

Because levels of participation in activities differed by age group and sex, we examined whether associations between activities and survival varied as a function of age and sex. We addressed this question by including interaction terms (age $\mathrm{x}$ activity category, sex $\mathrm{x}$ activity category) in the regression models. None of the interaction terms reached statistical significance (all $\mathrm{p}>.05$ ) in the analyses adjusted for the set of covariates, suggesting that associations between activities and survival were similar across age and for women and men. Consequently, we omit age- and sex-specific analyses and report only results using data from the full sample $(n=473)$.

Figure 2 about here

\section{Activities and Mortality Risk}

To test the association of everyday activities and mortality risk we proceeded in two stages. We first determined the zero-order relationships, and in a second step we evaluated mortality risks adjusted for the set of covariates (see Table 2). All activity types were significantly associated with risk of death in the unadjusted analyses. Higher levels of 
regenerative activities and lower levels of productive and consumptive activities were associated with an increased mortality risk. The magnitude of the associations was considerably reduced when we controlled for potential confounds and other activities. Only consumptive activities continued to be significantly $(\mathrm{p}<.05)$ associated with survival. The risk of death was reduced by $24 \%$ for individuals whose time spent on consumptive activities was above the median.

Table 2 about here

\section{Decreasing Returns on Investment}

We examined the hypothesis of decreasing returns with the aid of four strata reflecting the amount of time spent on an activity. All of the unadjusted relative risks increased or decreased monotonically with increasing daily activity duration (see Table 3 ). The more time spent on regenerative activities, the higher the risk of death. Similarly, the more time spent on productive and consumptive activities, the lower the unadjusted mortality risk.

After controlling for covariates, the effects associated with regenerative and productive activities disappeared, as was the case in the first set of analyses (Table 2). The pattern of adjusted risks that emerged for consumptive activities, however, is consistent with the hypothesis that a medium amount of time devoted to this activity is associated with the most beneficial effect: The third quarter of the activity distribution was associated with a lower risk than the other quarters. 
Table 3 about here

\section{DISCUSSION}

Time spent performing daily activities was related to mortality risk in this sample of persons aged 70 and older. In the unadjusted analyses, daily activity durations carried variance associated with common predictors of differential activity involvement and mortality risk. Above and beyond these confounding influences, only consumptive activities were associated with a reduced risk of death. When activity durations were grouped into four strata, there was some evidence of a non-linear relationship between activity duration and mortality risk, suggesting that a medium duration is related to the lowest risk.

\section{Consumptive Activities Linked with Survival}

Consistent with our hypothesis, consumptive activities were related to survival. This result lends support to the proposal that there is a psychosocial pathway linking daily activities and survival. We consider it likely that this psychosocial pathway involves perceived quality of life and subjective well-being. Consumptive activities affect perceived quality of life (Lawton et al. 1986-87), and they afford opportunities for aspiring, experience of competence, and autonomy. In contrast to some productive activities such as household chores, consumptive activities are more challenging and the demands they place can be more easily adjusted to one's skill level or preferences because they are more freely chosen. Moreover, they yield more feedback and, on average, more time is devoted to them per day. Engagement in consumptive activities may thus affect mental and physical health and, ultimately, survival.

\section{Productive Activities Not Linked with Survival}

Time spent on productive activities was not related to longevity in the adjusted analyses. It seems that decreasing time spent on productive activities (and also increasing time 
spent on regenerative activities) is not directly linked to mortality but rather is a marker of physical and mental decline. The lack of an association between productive activities and survival is not in accord with previous reports (e.g., Glass et al. 1999; Musick et al. 1999) positing that productive activities are linked with a longer life. One has to keep in mind, however, that in the present study the majority of productive activities were household chores - participation in paid and volunteer work was low in this sample (cf. Table 1; Klumb and Baltes 1999a). Household chores differ from other productive activities such as helping others or paid work. One difference is that—at least for people who live on their own-there are no others benefiting from the activity. Thus, household chores may often lack a characteristic of other productive activities, namely, confirming feedback and social recognition from others (Herzog et al. 1989; Herzog and Morgan 1992).

This discrepant finding may also have resulted from the fact that other researchers (e.g., Glass et al. 1999) employed younger samples and represented activity involvement by frequency ratings instead of time-use information. Frequency ratings are known to have two related disadvantages. First, it is possible to give high ratings for all cued activities because there is no frame of reference constraining the reports. In contrast, in a time budget the relationships between durations of different activities tend to be negative, because engagement in one type of activity typically precludes engagement in other activities at the same time. Secondly, frequency ratings are prone to third-variable confounding. Inter-individual differences in personality may lead to differential activity reports and these personality differences may themselves be associated with health and longevity (Chen and Spector 1991). Etiologic Mechanisms of the Psychosocial Pathway

Our results suggest that consumptive but not productive activities are associated with survival. This very pattern of results indicates that psychosocial mechanisms are involved which operate independently of the physical pathway. Note that about $60 \%$ of the time spent 
on consumptive activities in this sample consisted of purely sedentary activities such as reading or watching television (cf. Table 1), while most productive activities required at least some physical exertion. If the effect were driven solely by low-intensity physical activity (Riddoch 2000) then it should have been observed not only for consumptive but also for productive activities.

Which psychosocial processes can account for the link between daily activities and a longer life? It is likely that at least three different mechanisms are involved. First, performing challenging activities leads to the experience of competence, which is in turn related to wellbeing (Baltes et al. 1999; Karasek and Theorell 1990; White 1959). Second, social integration may enhance an individual's sense of meaning and purpose, which is related to well-being (Thoits 1983). Third, it is likely that the cognitive stimulation induced by challenging activities has beneficial effects on physical and mental health (Schooler, Mulatu, and Oates 1999; Wilson et al. 2002).

\section{Decreasing Returns on Investment}

We obtained some support for the idea that survival benefits do not increase monotonically with increasing involvement in consumptive activities. This is in line with research on self-reported health in which the marginal returns on active and passive leisure were found to decrease steadily, eventually becoming negative (Bird and Fremont 1991). Because of the small size of the sample utilized in the present study, some of the confidence intervals were relatively wide, and firm conclusions cannot be drawn here. Nevertheless we believe that the issue of decreasing returns deserves further attention. Investigations into this area will benefit from the increased statistical power afforded by larger samples and from attention to the duration of single episodes.

\section{Strengths and Limitations}


A strong point of this study is that we employed a theory-guided activity categorization. Furthermore, we used a well-defined sample which was stratified by age and sex and included a considerable number of very old persons. Assessment of activity involvement based on the Yesterday Interview yielded reliable and valid activity information with a meaningful metric and the advantageous characteristics of time-budget data. As covariates we rigorously chose common determinants of activity involvement and mortality from the data protocol of the Berlin Aging Study in order to reduce the confounding effects of third variables. The chosen covariates were based not only on participants' self-reporting but also on performance tests and physician-observed diagnoses of illnesses. This selection of covariates minimized confounding through common method variance.

In addition to the small sample size, at least three limitations should be kept in mind. First, we employed only activity data from a single day, and this day was not necessarily a typical one for all of the participants. It is thus likely that we underestimated the true size of the effects. Secondly, data did not contain sufficient information about the energy expenditure associated with performing an activity. Thus it was not possible to rigorously control for variance in physical exertion, although this would be have been desirable (Riddoch 2000). Finally, it is obvious that causal effects of activities on survival cannot be inferred from this study, even though activities preceded survival outcomes and activity involvement was associated with mortality risk after controlling for potential confounds. A risk factor can be called causal only if its manipulation changes the outcome (Kraemer et al. 1997), but we did not manipulate activity involvement in this study. However, the effects of altered engagement in consumptive activity can in principle be investigated in future intervention studies, because time spent on consumptive activities is malleable and amenable to intervention. We suggest that consumptive activities may contribute considerably to the maintenance of health and to 
longevity, because they are performed on a daily basis and their effects may accumulate over the life course.

\section{Conclusion}

Using time-budget data and a grouping of daily activities based on microeconomic theory, we found that consumptive activities were related to survival in persons aged 70 and older. This result supports psychological and sociological theorizing on the idea that daily activities and survival are linked through a psychosocial pathway, perhaps involving perceived quality of life, social recognition, and a sense of meaning and purpose. Consumptive activities may prove to be a promising target for intervention because they are performed on a daily basis. Future research on activity engagement and survival may benefit from adopting the concept of decreasing returns on investment. 
*We thank Hayden Bosworth, Karl Brehmer, Harvey Brenner, and Ursula Staudinger for their helpful comments.

The present research was conducted in the context of the Berlin Aging Study (BASE), which is co-chaired by Paul B. Baltes and Karl Ulrich Mayer. BASE has been financially supported by several organizations, including the German Federal Ministry for Research and Technology (1989-1991, 13 TA $011+13$ TA 011/A) and the German Federal Ministry for Family, Senior Citizens, Women, and Youth (1992-1998, 314-1722-102/9 + 314-1722102/9a).

Address correspondence to Petra L. Klumb, Public Health, BH 8, TU Berlin, ErnstReuter-Platz 1, 10587 Berlin, Germany. e-mail: petra.klumb@tu-berlin.de 


\section{REFERENCES}

Allison, P.D. 1995. Survival analysis using the SAS system: A practical guide. Cary, NC: SAS Institute.

Baltes, Margret M. 1996. “Successful aging.” Pp. 162-168 in Epidemiology in old age, edited by S. Ebrahim and A. Kalache. London: BMJ Publishing Group.

Baltes, Margret M., Ineke Maas, Hans-Ulrich Wilms, Markus Borchelt, and Todd Little. 1999. "Everyday competence in old and very old age: Theoretical considerations and empirical findings." Pp. 384-402 in The Berlin Aging Study: Aging from 70 to 100, edited by Paul B. Baltes and Karl Ulrich Mayer. New York: Cambridge University Press.

Baltes, Paul B., and Karl U. Mayer, eds. 1999. The Berlin Aging Study: Aging from 70 to 100. New York: Cambridge University Press.

Bird, Chloe E. , and Allen M. Fremont. 1991. “Gender, time use, and health.” Journal of Health and Social Behavior 32: 114-129.

Chen, P.Y., and P.E. Spector. 1991. "Negative affectivity as the underlying cause of correlations between stressors and strains." Journal of Applied Psychology 76: 398407.

Christensen, Kaare, and James W. Vaupel. 1996. "Determinants of longevity: genetic, environmental, and medical factors." Journal of Internal Medicine 240: 333-341.

Clark, Lee A., and David Watson. 1988. "Mood and the mundane: Relations between daily life events and self-reported mood." Journal of Personality and Social Psychology 54: 296-308.

Cohen, J. 1960. “A coefficient of agreement for nominal scales.” Educational and psychological measurement 20: 37-46. 
Cox, D.R. 1972. "Regression models and life tables." Journal of the Royal Statistical Society B34: 187-220.

Glass, T. A., C. M. de Leon, R. A. Marottoli, and L. F. Berkman. 1999. "Population based study of social and productive activities as predictors of survival among elderly Americans.” British Medical Journal 319: 478-483.

Goldberg, L., and D. L. Elliot. 1985. "The effect of physical activity on lipid and lipoprotein levels.“" Medical Clinics of North America 69: 41-55.

Helmert, U., B. Herman, and S. Shea. 1994. "Moderate and vigorous leisure-time physical activity and cardiovascular-disease risk-factors in West-Germany, 1984-1991.“ International Journal of Epidemiology 23: 285-292.

Herzog, A. R., R. L. Kahn, J. N. Morgan, J. S. Jackson, and T. C. Antonucci. 1989. “Age differences in productive activities." Journal of Gerontology: Social Sciences 44: 129138.

Herzog, A. R., and J. N. Morgan. 1992. "Age and gender differences in the value of productive activities." Research on Aging 14: 169-198.

House, J. S., K. L. Landis, and D. Umberson. 1988. “Social relationships and health.” Science 241: $540-545$.

Juster, F. T., and F. P. Stafford. 1991. "The allocation of time: Empirical findings, behavioral models, and problems of measurement." Journal of Economic Literature 29: 471-522.

Karasek, R. A., and Törres Theorell. 1990. Healthy work. New York: Basic Books. Klumb, Petra L., and Margret M. Baltes. 1999a. "Time use of old and very old Berliners: Productive and consumptive activities as functions of resources." Journal of Gerontology: Social Sciences 54B: S271-S278.

Klumb, Petra L., and Margret M. Baltes. 1999b. "Validity of retrospective time-use reports in old age." Applied Cognitive Psychology 13: 527-539. 
Kraemer, Helena Chmura, Alan E. Kazdin, David R. Offord, Ronald C. Kessler, Peter S. Jensen, and David J. Kupfer. 1997. "Coming to terms with the terms of risk." Archives of General Psychiatry 54: 337-343.

Lawton, M. P., M. Moss, and M. Fulcomer. 1986-87. "Objective and subjective uses of time by older people." International Journal of Aging and Human Development 24: 171188.

Lennartsson, Carin, and Merril Silverstein. 2001. "Does engagement with life enhance survival of elderly people in Sweden? The role of social and leisure activities." Journals of Gerontology: Social Sciences 25: S335--S342.

Lindenberger, U., R. Gilberg, T. Little, R. Nuthmann, U. Pötter, and P.B. Baltes. 1999. "Sample selectivity and generalizability of results of the Berlin Aging Study." Pp. 5682 in The Berlin Aging Study: Aging from 70 to 100, edited by P.B. Baltes and K.U. Mayer. New York: Cambridge University Press.

Moss, M. S., and M. P. Lawton. 1982. "Time budgets of older people: A window on four lifestyles." Journal of Gerontology 37: 115-123.

Musick, Marc A., A. Regula Herzog, and James S. House. 1999. "Volunteering and mortality among older adults: Findings from a national sample.” Journal of Gerontology: Social Sciences 54B: S173-S180.

Reid, M. 1934. Economics of household production. New York: Wiley.

Riddoch, C. 2000. "Social and productive activities in elderly people - Activities have been confused." British Medical Journal 320: 184.

Rowe, John W., and Robert L. Kahn. 1998. Successful Aging. New York: Pantheon Books.

Schooler, Carmi, Mesfin S. Mulatu, and Gary Oates. 1999. “The continuing effects of substantively complex work on the intellectual functioning of older workers." Psychology and Aging 14: 483-506. 
Thoits, Peggy A. 1983. "Multiple identies and psychological well-being: A reformulation and test of the social isolation hypothesis." American Sociological Review 48: 174-187.

Tinetti, M. E. 1986. “A performance-orientated assessment of mobility problems in elderly patients." Journal of the American Geriatrics Society 34: 119-126.

White, R. W. 1959. "Motivation considered: The concept of competence.” Psychological Review 66: 297-333.

Wilson, Robert S., Carlos F. Mendes de Leon, Lisa L. Barnes, Julie A. Schneider, Julia L. Bienias, Denis A. Evans, and David A. Bennett. 2002. "Participation in cognitively stimulating activities and risk of Alzheimer disease." Journal of the American Medical Association 287: 742-748. 
Table 1

Average time allocated to 3 activity categories and 13 activity domains $(\mathrm{N}=473)$.

\begin{tabular}{|c|c|c|}
\hline Activity category & Duration in minutes & $\%$ of the waking day \\
\hline Activity domain & $\underline{\mathrm{M}}(\underline{\mathrm{SD}})$ & $\underline{\mathrm{M}}(\underline{\mathrm{SD}})$ \\
\hline Regeneration & $330(177)$ & $34.6(19.1)$ \\
\hline Resting & $179(168)$ & $18.8(17.8)$ \\
\hline Self-maintenance & $151(58)$ & $15.8(6.2)$ \\
\hline Production & $150(130)$ & $15.5(13.1)$ \\
\hline Gardening & $13(45)$ & $1.3(4.4)$ \\
\hline Helping others & $3(18)$ & $0.3(1.9)$ \\
\hline Housework & $107(98)$ & $11.0(10.1)$ \\
\hline Paid work & $8(51)$ & $0.8(5.0)$ \\
\hline Running errands & $21(40)$ & $2.1(4.1)$ \\
\hline Consumption & $427(175)$ & $48.5(17.3)$ \\
\hline Active leisure & $83(103)$ & $8.4(10)$ \\
\hline Health-related activities & $9(21)$ & $0.9(2.1)$ \\
\hline Locomotion & $44(57)$ & $4.5(5.8)$ \\
\hline Social interaction & $63(81)$ & $6.6(8.8)$ \\
\hline Reading & $93(96)$ & $9.7(10.0)$ \\
\hline Watching TV/listening to & $179(135)$ & $18.4(13.8)$ \\
\hline
\end{tabular}


Table 2

Mortality risk associated with daily activities and with covariates $(\mathrm{N}=473)$.

Mortality risk

Risk factor

Unadjusted $^{\mathrm{a}}$

Adjusted $^{\mathrm{b}}$

$\begin{array}{lll}\text { Regenerative }(0=\text { low }, 1=\text { high }) & 2.04(1.63 \text { to } 2.56)^{* *} & 1.07(0.82 \text { to } 1.40) \\ \text { Productive }(0=\text { low, } 1=\text { high }) & 0.61(0.49 \text { to } 0.76)^{* *} & 0.96(0.74 \text { to } 1.25) \\ \text { Consumptive }(0=\text { low, } 1=\text { high }) & 0.59(0.47 \text { to } 0.74)^{* *} & 0.76(0.58 \text { to } 1.00)^{*}\end{array}$

Age (years)

$1.11(1.10 \text { to } 1.13)^{* *} \quad 1.08(1.06 \text { to } 1.10)^{* *}$

$\operatorname{Sex}(0=F, 1=M)$

1.39 (1.11 to 1.73$)^{* *}$

1.80 (1.38 to 2.35$)^{* *}$

Education (years)

0.98 (0.93 to 1.03$)$

1.01 (0.96 to 1.07$)$

Diagnoses (number)

$1.08(1.05 \text { to } 1.11)^{* *}$

$1.07(1.04 \text { to } 1.10)^{* * *}$

Balance / Gait (z-score) ${ }^{\mathrm{c}}$

$0.52(0.48 \text { to } 0.58)^{* *}$

$0.79(0.69 \text { to } 0.92)^{*}$

Digit Letter (z-score) $)^{\mathrm{c}}$

$0.57(0.51 \text { to } 0.64)^{* *}$

$0.92(0.80$ to 1.06$)$

With spouse $(0=\mathrm{N}, 1=\mathrm{Y})$

0.75 (0.59 to 0.96$)^{*}$

$1.02(0.75$ to 1.38$)$

In institution $(0=\mathrm{N}, 1=\mathrm{Y})$

$2.64(1.98 \text { to } 3.51)^{* *}$

$1.43(1.03 \text { to } 1.97)^{*}$

Note. Relative risks are reported. 95\% Confidence intervals for relative risks are shown in parentheses.

$* \mathrm{p}<.05, * * \mathrm{p}<.01$.

a Zero-order associations of each risk factor with mortality.

b Associations of risk factors with mortality after statistically controlling for all other factors. c Higher scores indicate higher levels of functioning. 


\section{Table 3}

Quarters of daily activities and mortality risk $(\mathrm{N}=473)$.

\begin{tabular}{|c|c|c|c|c|c|c|}
\hline \multirow[b]{2}{*}{ Activity Quarter } & \multicolumn{2}{|c|}{ Unadjusted } & \multicolumn{2}{|c|}{ Adjusted for covariates ${ }^{a}$} & \multicolumn{2}{|c|}{$\begin{array}{c}\text { Adjusted for covariates } \\
\text { and other activities }\end{array}$} \\
\hline & $\mathrm{RR}$ & $95 \% \mathrm{CI}$ & $\mathrm{RR}$ & $95 \% \mathrm{CI}$ & RR & $95 \% \mathrm{CI}$ \\
\hline \multicolumn{7}{|l|}{ Regenerative } \\
\hline$<25^{\text {th }} \%$ & 1 & & 1 & & 1 & \\
\hline$\geq 25^{\text {th }}$ and $<50^{\text {th }} \%$ & 1.35 & 0.96 to 1.90 & 0.90 & 0.63 to 1.28 & 0.90 & 0.63 to 1.29 \\
\hline$\geq 50^{\text {th }}$ and $<75^{\text {th }} \%$ & 1.66 & 1.19 to $2.32 * *$ & 1.01 & 0.70 to 1.44 & 0.95 & 0.65 to 1.38 \\
\hline$\geq 75^{\text {th }} \%$ & 3.55 & 2.58 to $4.88 * *$ & 1.24 & 0.87 to 1.78 & 1.14 & 0.75 to 1.73 \\
\hline \multicolumn{7}{|l|}{ Productive } \\
\hline$<25^{\text {th }} \%$ & 1 & & 1 & & 1 & \\
\hline$\geq 25^{\text {th }}$ and $<50^{\text {th }} \%$ & 0.63 & 0.47 to $0.85 * *$ & 1.21 & 0.88 to 1.65 & 1.25 & 0.91 to 1.72 \\
\hline$\geq 50^{\text {th }}$ and $<75^{\text {th }} \%$ & 0.55 & 0.40 to $0.74 * *$ & 1.15 & 0.82 to 1.62 & 1.18 & 0.83 to 1.68 \\
\hline$\geq 75^{\text {th }} \%$ & 0.41 & 0.30 to $0.57 * *$ & 1.13 & 0.79 to 1.63 & 1.10 & 0.73 to 1.68 \\
\hline \multicolumn{7}{|l|}{ Consumptive } \\
\hline$<25^{\text {th }} \%$ & 1 & & 1 & & 1 & \\
\hline$\geq 25^{\text {th }}$ and $<50^{\text {th }} \%$ & 0.81 & 0.60 to 1.08 & 0.98 & 0.72 to 1.32 & 0.99 & 0.71 to 1.36 \\
\hline$\geq 50^{\text {st }}$ and $<75^{\text {th }} \%$ & 0.55 & 0.40 to $0.75^{* *}$ & 0.71 & 0.51 to $0.99 *$ & 0.74 & 0.51 to 1.08 \\
\hline$\geq 75^{\text {th }} \%$ & 0.51 & 0.37 to $0.70^{* *}$ & 0.78 & 0.56 to 1.08 & 0.83 & 0.54 to 1.25 \\
\hline
\end{tabular}

Note. $\mathrm{RR}=$ Relative risk.

$\mathrm{CI}=$ Confidence interval

$* \mathrm{p}<.05, * * \mathrm{p}<.01$

a Adjusted mortality risks were obtained from Cox regression models including the respective activity, age, sex, education, number of diagnoses, balance / gait, digit letter, living with spouse, and living in institution. b Adjusted mortality risks were obtained from Cox regression models including all activity quarters, age, sex, education, number of diagnoses, balance / gait, digit letter, living with spouse, and living in institution. 


\section{Figure Captions}

Figure 1. A theory-based categorization of all activities into regenerative, productive, and consumptive activities.

Figure 2. Average time allocated to regenerative, productive, and consumptive activities (in minutes) for women and men aged 70-84 and 85+. 


\section{All activities during waking day}

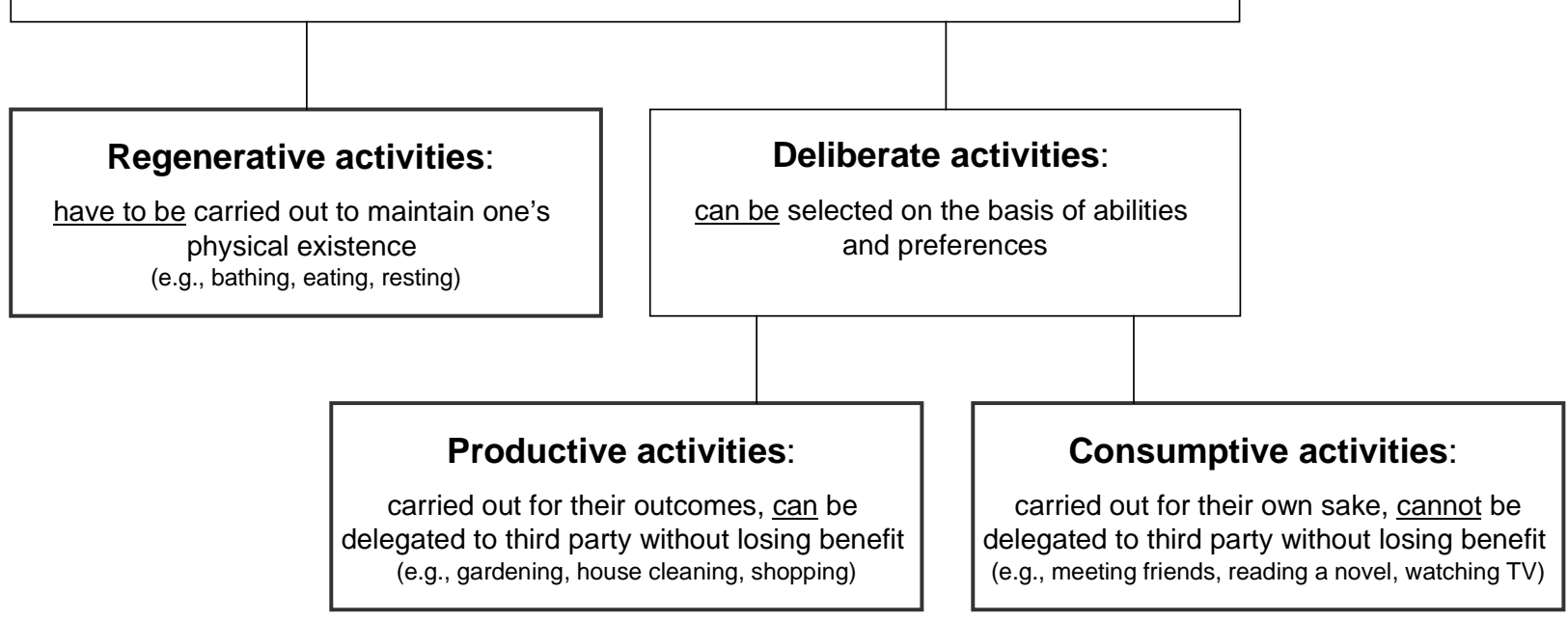




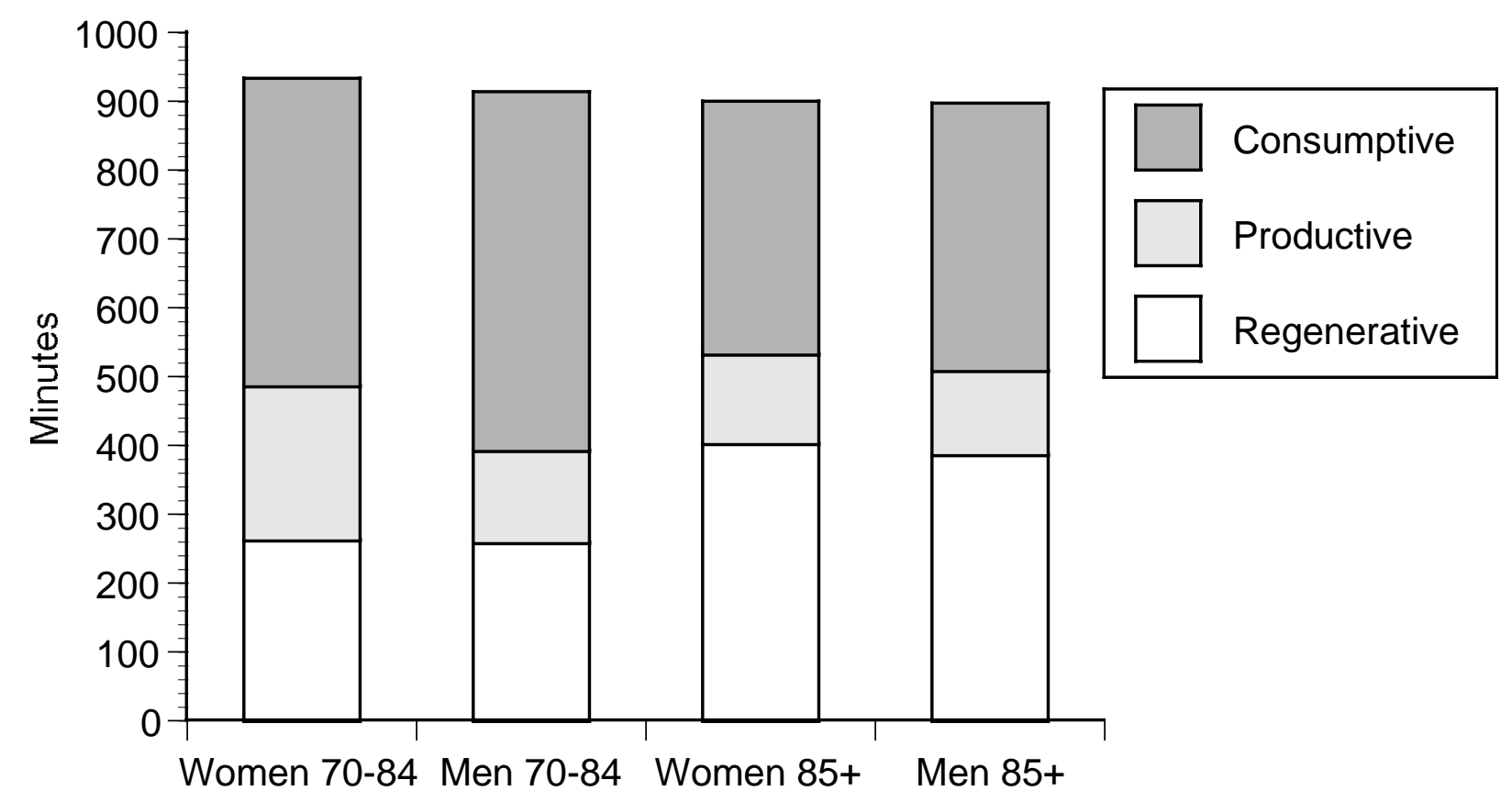

